\title{
Comportamento e preferências alimentares de pacientes com obesidade encaminhados à cirurgia bariátrica
}

\author{
Behavior and food preferences of obese patients referred to bariatric surgery \\ Jessica Santos Costa $^{1}$, Maria Clara Andrade Vaz ${ }^{2}$, Nadjane Ferreira Damascena ${ }^{3}$, Mônica Leila Portela de \\ Santana ${ }^{4}$, Cláudia Daltro de Sousa ${ }^{5}$, Carla Daltro ${ }^{6 *}$ \\ ${ }^{1}$ Nutricionista. Universidade Federal da Bahia (UFBA), Especialização em Nutrição Clínica-UFBA; ${ }^{2}$ Estudante do Curso \\ de Nutrição da Escola de Nutrição da UFBA; ${ }^{3}$ Doutoranda da Escola de Nutrição da UFBA; ${ }^{4}$ Professora Doutora \\ da Escola de Nutrição da UFBA; ${ }^{5}$ Nutricionista do Núcleo de Tratamento e Cirurgia da Obesidade. Doutoranda da \\ Escola de Medicina da UFBA.; ${ }^{6}$ Professora Doutora da Escola de Nutrição da UFBA. Endocrinologista do Núcleo de \\ Tratamento e Cirurgia da Obesidade.
}

\begin{abstract}
Resumo
Introdução: obesidade é um problema de saúde pública e está relacionada com fatores genéticos, ambientais e comportamento alimentar. É uma doença de difícil controle e a cirurgia bariátrica tem surgido com uma terapêutica de sucesso para obesos graves. Objetivo: descrever o comportamento e preferências alimentares de pacientes obesos encaminhados à cirurgia bariátrica. Metodologia: estudo descritivo baseado em dados secundários de uma clínica especializada no tratamento da obesidade. Foram estudadas variáveis antropométricas, sociodemográficas e outras referentes ao comportamento e preferências alimentares. Para análise dos dados foram utilizados: estatística descritiva e qui-quadrado de Pearson, sendo considerados significantes valores de $p<0,05$. Resultados: foram avaliados 542 pacientes com média (desvio padrão) de idade e índice de massa corporal de $35,9(9,8)$ anos e $41,5(4,9) \mathrm{kg} / \mathrm{m}^{2}$ respectivamente, sendo $73,6 \%$ do sexo feminino. Observou-se que os pacientes tinham preferência por cereais e massas $(62,4 \%)$, seguido de carne e ovos $(58,1 \%)$. Destacou-se que adultos jovens realizavam menos o desjejum $(65,1 \% \times 75,4 \% ; p=0,011)$ e ingeriam maior volume de alimentos nas refeições $(90,7 \%$ x 81,1\%; $p=0,007)$ quando comparados aos mais velhos e que o comportamento de acordar para comer à noite foi mais frequente nos homens do que nas mulheres $(31,2 \% \times 21,6 \% ; p=0,029)$. Conclusão: o estudo mostrou que os grupos alimentares preferidos foram os cereais e massas seguidos de carne e ovos. Quanto ao comportamento alimentar foi observado influência do sexo e da idade dos indivíduos, o mesmo não acontecendo em relação ao grau de obesidade.

Palavras-chave: Obesidade. Cirurgia Bariátrica. Comportamento alimentar. Preferências alimentares.
\end{abstract}

\begin{abstract}
Introduction: obesity is a public health problem related to genetic, environmental and eating behaviour factors. It is difficult to control, and bariatric surgery has emerged as a successful treatment for the severely obese. Objective: to describe the behaviour and food preferences of obese patients referred to bariatric surgery. Methodology: descriptive study based on secondary data from a clinic specialized in the treatment of obesity. Anthropometric, sociodemographic and other variables related to behavioural and food preferences were studied. For data analysis, descriptive statistics and Pearson's chi-square were used, with $p<0.05$ being considered significant. Results: 542 patients with mean age (standard deviation) and body mass index of 35.9 (9.8) years and 41.5 (4.9) kg / $\mathrm{m}^{2}$ respectively were evaluated, $73.6 \%$ of which were female. It was observed that the patients preferred to eat cereals and pasta (62.4\%), followed by meat and eggs (58.1\%). It was highlighted that young adults had less breakfast $(65.1 \% \times 75.4 \% ; p=0.011)$ and ingested a greater volume of food at meals $(90.7 \% \times 81.1 \% ; p=0.007)$ when compared to older people and that the behaviour of waking up to eat at night was more frequent in men than in women $(31.2 \% \times 21.6 \% ; p=0.029)$. Conclusion: the study showed that the preferred food groups were cereals and pasta followed by meat and eggs. As for eating behaviour, the influence of the individuals' sex and age was observed, the same not happening in relation to the degree of obesity.

Keywords: Obesity. Bariatric Surgery. Feeding behavior. Food preferences.
\end{abstract}

\section{INTRODUÇÃO}

A obesidade é uma doença crônica considerada como um importante problema de saúde pública, especialmente em países em desenvolvimento, com aumento significativo da prevalência em diversas populações do mundo. Segundo a Organização Mundial de Saúde (OMS) aproxi-

Correspondente/Corresponding: *Carla Daltro-End.: Av. Araújo Pinho - no 32 - Canela. Cep: 14.110-150 - Salvador - BA - Br. - Tel.: 71 32837700. -E-mail: carlahcdaltro@gmail.com madamente dois bilhões de adultos apresentavam peso acima do recomendado em 2016 (WHO, 2016; ABESO, 2016). Estimativas recentes do Sistema de Vigilância de Fatores de Risco e Proteção para Doenças Crônicas por Inquérito Telefônico (VIGITEL) mostram que $55,7 \%$ dos adultos brasileiros tinham excesso de peso e $19,8 \%$ obesidade em 2018 (BRASIL. MINISTÉRIO DA SAÚDE, 2018).

O acúmulo excessivo de gordura corporal indica a presença da obesidade, favorecendo o desenvolvimento de várias comorbidades e distúrbios metabólicos, 
a exemplo de diabetes, doenças cardiovasculares, osteoartrites, e alguns tipos de cânceres (BRASIL. MINISTÉRIO DA SAÚDE, 2014; RODRIGUES et al., 2014). A OMS recomenda o Índice de Massa Corporal (IMC) como padrão internacional para o diagnóstico da obesidade e propõe pontos de corte de classificação do grau da obesidade em adultos, em grau I quando o valor do IMC estiver entre $30 \mathrm{~kg} / \mathrm{m}^{2}$ e $34,9 \mathrm{~kg} / \mathrm{m}^{2}$; grau II entre $35 \mathrm{~kg} /$ $\mathrm{m}^{2}$ e $39,9 \mathrm{~kg} / \mathrm{m}^{2}$; e grau III ou mórbida, se este índice for igual ou maior a $40 \mathrm{~kg} / \mathrm{m}^{2}$ (WHO, 2000). Evidências mostram que a obesidade é uma doença de etiologia multifatorial, influenciada por uma complexa interação entre fatores genéticos, ambientais, emocionais e do estilo de vida, que envolvem comportamentos alimentares (ABESO, 2016; SILVA; CRAHIM, 2019). Sugere-se que beliscar e ter preferência por consumir alimentos gordurosos tendem a aumentar de acordo com o grau de obesidade (BARRAGÁN et al., 2015). E, indivíduos com obesidade tinham comportamento alimentar não saudável, como hiperfagia noturna, comer quando não estavam com fome, e perda de controle ao comer, antes da realização da cirurgia bariátrica (COLLES; DIXON; O'BRIEN, 2007; MITCHELL et al., 2015).

Assim, diante do quadro apresentado, intensificado pela forte pressão pessoal e social, indivíduos com obesidade adotam estratégias e práticas de emagrecimento com ou sem o auxílio de profissional especializado (CARVALHO; VASCONCELOS; CARVALHO, 2016), o que provavelmente dificulta a perda ponderal, e principalmente a sustentação dela. Nesse cenário, indivíduos com obesidade mórbida adotam o procedimento cirúrgico como tratamento de eleição para reduzir os danos físicos e psicossociais (JESUS et al., 2017).

Contudo, apesar da cirurgia bariátrica ser uma intervenção considerada eficaz para o tratamento de pessoas com obesidade mórbida, para um subgrupo de pacientes o período pós-operatório pode estar associado à redução do peso insatisfatória ou inexistente e ao reganho ponderal significativo. Diversos fatores podem influenciar esses desfechos pós cirurgia, a exemplo dos comportamentos alimentares inadequados (MITCHELL et al., 2015). Dessa forma, o objetivo desse estudo foi descrever o comportamento e as preferências alimentares de pacientes obesos encaminhados à cirurgia bariátrica.

\section{METODOLOGIA}

Trata-se de um estudo descritivo com pacientes atendidos no Núcleo de Tratamento e Cirurgia da Obesidade (NTCO) entre 2014 e 2016 na cidade de Salvador-Bahia. O NTCO é um centro para tratamento multidisciplinar da obesidade que atende pacientes particulares e conveniados. No protocolo de atendimento do NTCO é feita avaliação pré-operatória dos pacientes por nutricionistas, médicos, psicólogos, fisioterapeutas, fonoaudiólogos, além de educador físico e assistente social. Para compor este estudo, foram utilizados dados secundários provenientes dos prontuários dos pacientes no primeiro atendimento que continha informações de um questionário autoaplicado na consulta inicial para a cirurgia bariátrica, de acordo com o protocolo de atendimento do serviço.

Os pacientes com obesidade graus II e III (IMC $\geq 35$ $\mathrm{Kg} / \mathrm{m}^{2}$ ) e com idade superior a 18 anos foram considerados elegíveis para este estudo. Não foram incluídos aqueles com insuficiência cardíaca, hepática, pancreática, renal, respiratória que pudessem afetar de maneira importante a composição corporal e pacientes que apresentavam disfunção tireoidiana descompensada.

Os dados antropométricos, peso e altura, registrados no prontuário foram utilizados para calcular o IMC e determinar o grau da obesidade com base nos pontos de corte propostos pela OMS. De acordo com esses pontos de corte, considera-se obesidade grau II quando o indivíduo tem o IMC entre $35 \mathrm{~kg} / \mathrm{m}^{2}$ e $39,9 \mathrm{~kg} /$ $\mathrm{m}^{2}$ e grau III ou mórbida se o valor de IMC for igual ou maior a $40 \mathrm{~kg} / \mathrm{m}^{2}$ (WHO, 2000). A altura foi aferida em estadiômetro portátil com graduação de $1 \mathrm{~mm}$, e o peso foi obtido na bioimpedância segundo protocolo indicado pelo fabricante do equipamento (Biospace ${ }^{\circledR}$ ).

Um questionário estruturado e autoaplicado utilizado na consulta inicial no NTCO continha informações sociodemográficas (idade, sexo, escolaridade, estado civil), do comportamento alimentar (número de fracionamento das refeições, realização do desjejum, turno da fome, acordar para comer à noite, comer volume excessivo, sentir-se mal após comer volumes excessivos de alimentos, "beliscar alimentos", adoção de vômito autoinduzido por se sentir culpado após comer) e das preferências alimentares.

O fracionamento das refeições se referiu à frequência de refeições que o participante fazia ao dia, incluindo lanches, e teve as respostas estratificadas em menos que 3 refeições por dia, entre 3 e 4, entre 5 e 6 e maior que 6. 0 desjejum foi considerado como a ingestão de alimentos na primeira refeição da manhã, entre 4 e 11 horas (MONTEIRO et al, 2017). A resposta para esta variável foi categorizada em "sim" ou "não". O turno da fome consistiu em qual horário do dia que sentia maior apetite e a resposta foi estratificada em manhã, tarde e noite.

Comer no período noturno significou acordar durante a noite para fazer a ingestão de alimentos. Comer volume excessivo de alimentos ficou a critério de cada integrante sobre o que entendia por volume excessivo por refeição. A questão sentir-se mal após comer volumes excessivos de alimentos se referiu à possíveis sintomas como dor ou desconforto que o paciente sentia ao comer grandes volumes de alimentos. A variável "beliscar alimentos" indicou fazer pequenas refeições várias vezes durante o dia. A informação sobre vômito autoinduzido consistiu em provocar o vômito por se sentir culpado após comer. As respostas para essas cinco questões eram compostas de sempre, às vezes e nunca. 
Foi solicitado a cada participante que anotasse seus alimentos preferidos no questionário estruturado autoaplicado preenchido na primeira consulta. Após isso foi segregado cada alimento de acordo com os grupos alimentares, tendo como base o Guia alimentar para população brasileira (BRASIL. MINISTÉRIO DA SAÚDE, 2008).

Para a tabulação e análise dos dados foi utilizado o programa estatístico SPSS for Windows ${ }^{\circledR}$ 20.0. Os resultados das variáveis contínuas foram apresentados sob a forma de média e desvio padrão (DP) e as variáveis categóricas foram expressas por frequência absoluta e relativa. Para a comparação das variáveis qualitativas foi utilizado o teste qui-quadrado de Pearson. Foram considerados como estatisticamente significantes valores de $p<0,05$.

Esse estudo foi aprovado pelo Comitê de Ética em Pesquisa da Escola de Nutrição da UFBA (parecer no 2.999.883, de 2018). Os pacientes do estudo assinaram o Termo de Consentimento Livre e Esclarecido (TCLE).

\section{RESULTADOS}

Foram selecionados 787 pacientes para participarem deste estudo, no entanto 245 foram excluídos por terem dados incompletos permanecendo ao final uma amostra de 542 indivíduos obesos. A maioria era do sexo feminino $(73,6 \%)$ e a idade variou entre 18 e 71 anos, com média (DP) igual a 35,9 $(9,8)$ anos. A média (DP) do IMC foi 41,5 $(4,9) \mathrm{kg} / \mathrm{m}^{2}$. Aproximadamente $50 \%$ dos indivíduos tinham o terceiro grau completo e $50,7 \%$ eram casados (Tabela 1 ).
Tabela 1 - Características sociodemográficas e antropométrica dos 542 pacientes com obesidade, atendidos no NTCO, entre 2014 e 2016, Salvador, Bahia.

\begin{tabular}{lr}
\hline \multicolumn{1}{c}{ Variáveis } & \multicolumn{1}{c}{ N (\%) } \\
\hline Sexo Feminino & $399(73,6)$ \\
Idade (anos)* $^{*}$ & $35,9(9,8)$ \\
Escolaridade** & \\
$\quad$ Analfabeto & $2(0,4)$ \\
$\quad$ Primeiro grau completo & $30(6,4)$ \\
Segundo grau completo & $205(43,5)$ \\
Terceiro grau completo & $234(49,7)$ \\
Estado civil** & \\
Solteiro & $239(44,8)$ \\
Casado & $271(50,7)$ \\
Separado & $16(3)$ \\
Viúvo & $8(1,5)$ \\
IMC (kg/ $\left.\mathrm{m}^{2}\right)^{*}$ & $41,5(4,9)$ \\
\hline
\end{tabular}

*Média (DP); ${ }^{* *}$ Dados incompletos de alguns pacientes.

Fonte: Dados da pesquisa

Na tabela 2 observa-se a distribuição das informações sobre o comportamento alimentar dos participantes de acordo com a idade, sexo e grau de obesidade. Quando comparado os adultos jovens com os mais velhos, observou-se que os primeiros realizavam menos o desjejum $(65,1 \% \times 75,4 \%, p=0,011)$ e ingeriam volume excessivo de comida com mais frequência que os mais velhos $(90,7 \% \mathrm{x}$ $81,1 \%, p=0,007)$. Em relação ao sexo, chama a atenção o fato dos homens mais frequentemente comerem no período noturno quando comparado com as mulheres $(31,2 \%$ $\times 21,6 \%, p=0,029$ ). A tabela 2 traz ainda informações sobre o comportamento alimentar de pacientes de acordo com o grau de obesidade, não sendo observado nenhuma diferença significativa entre as variáveis estudadas.

Tabela 2 - Comportamento alimentar de pacientes obesos de acordo com idade, sexo e grau de obesidade, atendidos no Núcleo de Tratamento e Cirurgia da Obesidade, Salvador, Bahia, entre 2014 e 2016.

\begin{tabular}{|c|c|c|c|c|c|c|c|c|c|c|}
\hline \multirow[t]{3}{*}{ Variáveis } & \multirow[t]{3}{*}{$\begin{array}{l}\text { Total } \\
\text { N (\%) }\end{array}$} & \multicolumn{2}{|c|}{ Idade } & \multirow{3}{*}{ p } & \multicolumn{2}{|c|}{ Sexo } & \multirow{3}{*}{$\mathbf{p}$} & \multicolumn{2}{|c|}{$\begin{array}{l}\text { Obesidade mórbida } \\
\left(\text { (IMC } \geq 40 \mathrm{~kg} / \mathrm{m}^{2}\right)\end{array}$} & \multirow{3}{*}{ p } \\
\hline & & $\begin{array}{c}<35 \text { anos } \\
\mathbf{N}(\%)\end{array}$ & $\begin{array}{c}\geq 35 \text { anos } \\
\quad \mathbf{N}(\%)\end{array}$ & & $\begin{array}{l}\text { Masculino } \\
\text { N (\%) }\end{array}$ & $\begin{array}{l}\text { Feminino } \\
\mathbf{N}(\%)\end{array}$ & & $\begin{array}{c}\text { Sim } \\
\mathbf{N}(\%)\end{array}$ & $\begin{array}{c}\text { Não } \\
\text { N (\%) }\end{array}$ & \\
\hline & & $253(46,7)$ & $289(53,3)$ & & $143(26,4)$ & $399(73,6)$ & & $296(54,6)$ & $246(45,4)$ & \\
\hline \multicolumn{11}{|c|}{$\begin{array}{l}\text { Fracionamento das refei- } \\
\text { ções/dia * }\end{array}$} \\
\hline Menor que 3 & $15(2,8)$ & $6(2,4)$ & $9(3,1)$ & 0,527 & $7(5,0)$ & $8(2,0)$ & 0,127 & $9(3,1)$ & $6(2,5)$ & 0,821 \\
\hline 3 a 4 & $194(36,4)$ & $84(34,0)$ & $110(38,5)$ & & $57(40,7)$ & $137(34,9)$ & & $108(37,2)$ & $86(35,4)$ & \\
\hline 5 a 6 & $241(45,2)$ & $120(48,6)$ & $121(42,3)$ & & $55(39,3)$ & $186(47,3)$ & & $126(43,4)$ & $115(47,3)$ & \\
\hline Maior que 6 & $83(15,6)$ & $37(15,0)$ & $46(16,1)$ & & $21(15,0)$ & $62(15,8)$ & & $47(16,2)$ & $36(14,8)$ & \\
\hline Desjejum* & $366(70,7)$ & $155(65,1)$ & $211(75,4)$ & 0,011 & $94(69,1)$ & $272(71,2)$ & 0,646 & $199(71,3)$ & $167(69,9)$ & 0,718 \\
\hline \multicolumn{11}{|l|}{ Turno da fome* } \\
\hline Manhã & $66(12,5)$ & $26(10,5)$ & $40(14,3)$ & 0,307 & $18(12,8)$ & $48(12,4)$ & 0,794 & $38(13,2)$ & $28(11,6)$ & 0,326 \\
\hline Tarde & $154(29,2)$ & $78(31,5)$ & $76(27,1)$ & & $38(27,0)$ & $116(30,0)$ & & $90(31,4)$ & $64(26,6)$ & \\
\hline Noite & $308(58,3)$ & $144(58,1)$ & $164(58,6)$ & & $85(60,3)$ & $223(57,6)$ & & $159(55,4)$ & $149(61,8)$ & \\
\hline
\end{tabular}


(Continuação)

\begin{tabular}{|c|c|c|c|c|c|c|c|c|c|c|}
\hline \multirow[t]{3}{*}{ Variáveis } & \multirow[t]{3}{*}{$\begin{array}{l}\text { Total } \\
\text { N (\%) }\end{array}$} & \multicolumn{2}{|c|}{ Idade } & \multirow{3}{*}{ p } & \multicolumn{2}{|c|}{ Sexo } & \multirow{3}{*}{ p } & \multicolumn{2}{|c|}{$\begin{array}{l}\text { Obesidade mórbida } \\
\left(I M C \geq 40 \mathrm{~kg} / \mathrm{m}^{2}\right)\end{array}$} & \multirow{3}{*}{ p } \\
\hline & & $\begin{array}{c}<35 \text { anos } \\
\mathrm{N}(\%)\end{array}$ & $\begin{array}{c}\geq 35 \text { anos } \\
N(\%)\end{array}$ & & $\begin{array}{l}\text { Masculino } \\
\text { N (\%) }\end{array}$ & $\begin{array}{l}\text { Feminino } \\
\mathrm{N}(\%)\end{array}$ & & $\begin{array}{l}\text { Sim } \\
\mathbf{N}(\%)\end{array}$ & $\begin{array}{c}\text { Não } \\
\text { N (\%) }\end{array}$ & \\
\hline & & $253(46,7)$ & $289(53,3)$ & & $143(26,4)$ & $399(73,6)$ & & $296(54,6)$ & $246(45,4)$ & \\
\hline \multicolumn{11}{|l|}{$\begin{array}{l}\text { Comer no período no- } \\
\text { turno * }\end{array}$} \\
\hline Sempre & $15(2,8)$ & $3(1,2)$ & $12(4,3)$ & 0,103 & $3(2,1)$ & $12(3,1)$ & 0,029 & $5(1,7)$ & $10(4,2)$ & 0,228 \\
\hline Às vezes & $113(21,3)$ & $54(21,6)$ & $59(21,0)$ & & $41(29,1)$ & $72(18,5)$ & & $64(22,0)$ & $49(20,4)$ & \\
\hline Nunca & $403(75,9)$ & $193(77,2)$ & $210(74,7)$ & & $97(68,8)$ & $306(78,5)$ & & $222(76,3)$ & $181(75,4)$ & \\
\hline \multicolumn{11}{|l|}{ Volume em excesso* } \\
\hline Sempre & $79(14,8)$ & $40(16,3)$ & $39(13,6)$ & 0,007 & $25(17,7)$ & $54(13,8)$ & 0,396 & $45(15,5)$ & $34(14,1)$ & 0,574 \\
\hline Às vezes & $376(70,7)$ & $183(74,4)$ & $193(67,5)$ & & $99(70,2)$ & $277(70,8)$ & & $208(71,5)$ & $168(69,7)$ & \\
\hline Nunca & $77(14,5)$ & $23(9,3)$ & $54(18,9)$ & & $17(12,1)$ & $60(15,3)$ & & $38(13,1)$ & $39(16,2)$ & \\
\hline \multicolumn{11}{|l|}{$\begin{array}{l}\text { Sentir-se mal após co- } \\
\text { mer* }\end{array}$} \\
\hline Sempre & $8(1,5)$ & $3(1,2)$ & $5(1,8)$ & 0,145 & $1(0,7)$ & $7(1,8)$ & 0,670 & $5(1,7)$ & $3(1,2)$ & 0,628 \\
\hline Às vezes & $177(33,3)$ & $93(37,5)$ & $84(29,6)$ & & $47(33,6)$ & $130(33,2)$ & & $92(31,6)$ & $85(35,3)$ & \\
\hline Nunca & $347(65,2)$ & $152(61,3)$ & $195(68,7)$ & & $92(65,7)$ & $255(65,1)$ & & $194(66,7)$ & $153(63,5)$ & \\
\hline \multicolumn{11}{|l|}{ Vômito autoinduzido* } \\
\hline Sempre & $5(0,9)$ & $1(0,4)$ & $4(1,4)$ & 0,158 & - & $5(1,3)$ & 0,331 & $3(1,0)$ & $2(0,8)$ & 0,965 \\
\hline Às vezes & $28(5,2)$ & $17(6,8)$ & $11(3,9)$ & & $6(4,7)$ & $22(5,6)$ & & $15(5,1)$ & $13(5,4)$ & \\
\hline Nunca & $502(93,8)$ & $232(92,8)$ & $270(94,7)$ & & $135(95,7)$ & $367(93,1)$ & & $275(93,4)$ & $227(93,8)$ & \\
\hline Beliscar alimentos* & $266(49,1)$ & $120(47,8)$ & $146(51,2)$ & 0,730 & $67(47,5)$ & $199(50,4)$ & 0,577 & $147(50,2)$ & $119(49,0)$ & 0,703 \\
\hline Sempre & $221(41,2)$ & $107(42,6)$ & $114(40,0)$ & & $63(44,7)$ & $158(40,0)$ & & $122(41,6)$ & $99(40,7)$ & \\
\hline Às vezes & $49(9,1)$ & $24(9,6)$ & $25(8,8)$ & & $11(7,8)$ & $38(9,6)$ & & $24(8,2)$ & $25(10,3)$ & \\
\hline Nunca & $15(2,8)$ & $6(2,4)$ & $9(3,1)$ & & $7(5,0)$ & $8(2,0)$ & & $9(3,1)$ & $6(2,5)$ & \\
\hline
\end{tabular}

*Dados incompletos de alguns pacientes.

Fonte: Dados da pesquisa

Os grupos de alimentos preferidos para o consumo pelos participantes estão distribuídos na tabela 3 . Independentemente da idade, sexo ou grau de obesidade, os grupos preferidos pela maioria dos pacientes foram cereais e massas seguido pelo de carnes e ovos.

Tabela 3 - Preferências alimentares de pacientes obesos de acordo com idade, sexo e grau de obesidade, atendidos no Núcleo de Tratamento e Cirurgia da Obesidade, Salvador, Bahia, entre 2014 e 2016.

\begin{tabular}{|c|c|c|c|c|c|c|c|c|c|c|}
\hline \multirow{3}{*}{$\begin{array}{l}\text { Preferência } \\
\text { alimentar }\end{array}$} & \multirow[t]{3}{*}{$\begin{array}{l}\text { Total } \\
\text { N (\%) }\end{array}$} & \multicolumn{2}{|c|}{ Idade } & \multirow{3}{*}{ p } & \multicolumn{2}{|c|}{ Sexo } & \multirow{3}{*}{$p$} & \multicolumn{2}{|c|}{$\begin{array}{c}\text { Obesidade mórbida } \\
\left(\mathrm{IMC} \geq 40 \mathrm{~kg} / \mathrm{m}^{2}\right)\end{array}$} & \multirow{3}{*}{ p } \\
\hline & & $\begin{array}{c}<35 \text { anos } \\
\quad \mathbf{N}(\%)\end{array}$ & $\begin{aligned} \geq & 35 \text { anos } \\
& \mathbf{N}(\%)\end{aligned}$ & & $\begin{array}{l}\text { Masculino } \\
\text { N (\%) }\end{array}$ & $\begin{array}{l}\text { Feminino } \\
\text { N (\%) }\end{array}$ & & $\begin{array}{l}\text { Sim } \\
\text { N (\%) }\end{array}$ & $\begin{array}{l}\text { Não } \\
\text { N (\%) }\end{array}$ & \\
\hline & & $\begin{array}{c}253 \\
(46,7)\end{array}$ & $\begin{array}{c}289 \\
(53,3)\end{array}$ & & $\begin{array}{c}143 \\
(26,4)\end{array}$ & $\begin{array}{c}399 \\
(73,6)\end{array}$ & & $\begin{array}{c}296 \\
(54,6)\end{array}$ & $\begin{array}{c}246 \\
(45,4)\end{array}$ & \\
\hline Cereais e massas & $338(62,4)$ & $152(60,1)$ & $186(64,4)$ & 0,305 & $85(59,4)$ & $253(63,4)$ & 0,401 & $182(61,5)$ & $156(63,4)$ & 0,645 \\
\hline Carne e ovos & $315(58,1)$ & $157(62,1)$ & $158(54,7)$ & 0,082 & $87(60,8)$ & $228(57,1)$ & 0,442 & $163(55,1)$ & $152(61,8)$ & 0,114 \\
\hline Leguminosas & $105(19,4)$ & $48(19,0)$ & $57(19,7)$ & 0,825 & $28(19,6)$ & $77(19,3)$ & 0,942 & $59(19,9)$ & $46(18,7)$ & 0,718 \\
\hline Frutas & $112(20,7)$ & $47(18,6)$ & $65(22,5)$ & 0,262 & $33(23,1)$ & $79(19,8)$ & 0,406 & $66(22,3)$ & $46(18,7)$ & 0,303 \\
\hline Legumes & $83(15,3)$ & $43(17,0)$ & $40(15,8)$ & 0,309 & $20(14,0)$ & $63(15,8)$ & 0,607 & $47(15,9)$ & $36(14,6)$ & 0,689 \\
\hline Açúcares & $60(11,1)$ & $30(11,9)$ & $30(10,4)$ & 0,584 & $20(14,0)$ & $40(10,0)$ & 0,195 & $36(12,2)$ & $24(9,8)$ & 0,374 \\
\hline Verduras & $49(9,0)$ & $23(9,1)$ & $26(9,0)$ & 0,970 & $12(8,4)$ & $37(9,3)$ & 0,752 & $23(7,8)$ & $26(10,6)$ & 0,258 \\
\hline Leite e derivados & $46(8,5)$ & $22(8,7)$ & $24(8,3)$ & 0,871 & $14(9,8)$ & $32(8,0)$ & 0,515 & $22(7,4)$ & $24(9,8)$ & 0,334 \\
\hline Comidas típicas & $23(4,2)$ & $14(5,5)$ & $9(3,1)$ & 0,163 & $5(3,5)$ & $18(4,5)$ & 0,606 & $12(4,1)$ & $11(4,5)$ & 0,810 \\
\hline Gorduras & $26(4,8)$ & $13(5,1)$ & $13(4,5)$ & 0,728 & $6(1,2)$ & $20(5,0)$ & 0,695 & $14(4,7)$ & $12(4,9)$ & 0,936 \\
\hline Raízes e tubérculos & $8(1,5)$ & $4(1,6)$ & $4(1,4)$ & 0,850 & $2(1,4)$ & $6(1,5)$ & 0,929 & $2(0,7)$ & $6(2,4)$ & 0,090 \\
\hline
\end{tabular}

Fonte: Dados da pesquisa 


\section{DISCUSSÃO}

O presente estudo mostrou que os grupos alimentares preferidos foram os cereais e massas seguidos de carne e ovos. Quanto ao comportamento alimentar foi observado influência do sexo e da idade dos indivíduos, o mesmo não acontecendo em relação ao grau de obesidade.

Em relação à idade, observou-se que adultos jovens aderiram menos ao desjejum em relação a pessoas mais velhas. $O$ estudo publicado por Mitchell et al. (2015) investigou padrões alimentares antes da cirurgia bariátrica e encontrou que a maioria dos participantes relatou tomar café da manhã regularmente, porém não avaliou esses dados de acordo com a idade dos indivíduos. Nota-se que o café da manhã é uma das principais refeições do dia e realizar seu consumo tem relação direta com um padrão alimentar mais saudável (UZHOVA et al., 2018). O estudo de Uzhova et al. (2018), mostrou aumento do risco cardiovascular, perfil lipídico desfavorável, obesidade e diabetes com a não adesão ao desjejum. Nessa direção, resultados de outro estudo sugere ainda que a realização do café da manhã pode contribuir para regulação do apetite, aumentando a saciedade e, assim, reduzindo a ingestão de calorias diárias (PEREIRA et al., 2011).

Além disso, os adultos jovens também ingeriam maior volume de alimento em relação aos mais velhos, o que está em consonância com a literatura. OVED et al., (2017) chamam a atenção para a perda do controle da qualidade e da quantidade do alimento consumido ao longo do dia, no entanto não avaliaram essa relação de acordo com a idade dos indivíduos. Uma possível explicação para os nossos resultados é que as pessoas mais velhas, provavelmente já devem ter buscado outras estratégias para reduzir a quantidade de alimentos ingeridos, e nesse contexto, começaram a aderir refeições sem excessos. Em estudo recentemente conduzido, os autores concluíram que a ocorrência de compulsão alimentar antes da cirurgia bariátrica é prevalente e que esse comportamento ainda permanece, mesmo após 3 a 4 anos de cirurgia (RIBEIRO et al., 2018).

Quando questionados sobre o horário que sentiam mais fome, os pacientes indicaram o turno da noite, apesar da maioria não apresentar o comportamento de acordar para comer neste horário. Contudo, a maior frequência desse comportamento foi observada entre os adultos do sexo masculino. Anger e Katz (2015), em seu estudo com indivíduos com obesidade e na fila para cirurgia bariátrica, encontraram que uma quantidade expressiva dos indivíduos do sexo masculino apresentaram alimentação noturna em relação ao sexo feminino. Conforme Gallant, Lundgren e Drapeau (2012) é evidente que a prevalência do comer no período noturno seja maior em indivíduos com obesidade do que na população em geral e que a ingestão noturna está associada com excesso de calorias e consequentemente, com maior dificuldade para a perda e manutenção do peso.
Apesar de não ter sido observado nenhuma diferença significativa entre as variáveis dos grupos de alimentos preferidos e idade, sexo e grau de obesidade, destaca-se neste estudo uma preferência alimentar pelos grupos de cereais e massas seguido por carnes e ovos. Dados semelhantes foram descritos por Jastrzębska-Mierzyńska et al. (2014), que apesar de não estudarem os grupos alimentares, encontraram em seu trabalho que a maioria dos indivíduos consumia pão branco, pelo menos uma vez por dia, e macarrão e arroz, semanalmente. A principal fonte de proteína presente na dieta dos pacientes com obesidade era a carne, sendo as mais comumente consumidas as de frango e de porco. Neste estudo, apesar dos pacientes terem apresentado essa preferência alimentar, não significa que eles comam esses alimentos com maior frequência.

Podemos considerar algumas limitações neste estudo. A primeira diz respeito ao uso de dados secundários fornecidos por questionário utilizado para obter as informações de forma autorreferida na consulta inicial. No entanto são instrumentos desenvolvidos por nutricionistas com vasta experiência no atendimento pré e pós cirurgia bariátrica e os pacientes são alertados da importância desses dados para o grupo de especialistas que os conduzirão. A segunda limitação diz respeito a escassez de estudos na literatura sobre comportamento e preferências alimentares para que pudéssemos comparar com os nossos resultados; e por último, os dados de preferência alimentar poderiam ser coletados juntamente com um instrumento validado de inquérito alimentar. $O$ estudo também exibe alguns pontos fortes como possuir uma amostra grande de pacientes; tratar de um tema pouco abordado na literatura; contribuir e incentivar novas pesquisas nessa área.

\section{CONCLUSÃO}

Os resultados mostraram que pacientes adultos encaminhados à cirurgia bariátrica diferiram quanto as preferências e comportamentos alimentares quando analisados de acordo com o sexo e idade, o mesmo não sendo evidenciado em relação ao grau de obesidade. Adultos jovens realizavam menos o desjejum e ingeriam maior volume de alimentos, e os homens adotavam a prática de comer no período noturno mais frequentemente que as mulheres. Não foi observada diferença relativa aos grupos de alimentos preferidos, porém é importante destacar preferência maior por cereais e massas, seguido de carne e ovos.

Acreditamos que essas informações são importantes para construção de um plano alimentar individualizado, juntamente com um trabalho minucioso de educação nutricional como ferramenta auxiliar direcionada aos pacientes que buscam uma perda ponderal. 


\section{REFERÊNCIAS}

ANGER, V.; KATZ, M. Relación entre imc, emociones percibidas, estilo de ingesta y preferencias gustativas en una población de adultos. Actual. Nutr., Buenos Aires, v. 16, n. 1, p. 31-36, 2015.

ASSOCIAÇÃO BRASILEIRA PARA O ESTUDO DA OBESIDADE E DA SÍNDROME METABÓLICA (ABESO). Diretrizes Brasileiras de Obesidade 2015/2016. 4. ed. São Paulo: ABESO, 2016.

BARRAGÁN, A. M. R. B. et al. Hábitos de alimentación de pacientes con obesidad severa. Nutr. Hosp., Madrid, v. 31, n. 2, p. 672-681, 2015.

BRASIL. Ministério da Saúde. Secretaria de Atenção à Saúde. Guia alimentar para a população brasileira: promovendo a alimentação saudável. Brasília: Ministério da Saúde; 2008.

BRASIL. Ministério da Saúde. Sociedade Brasileira de Cirurgia Bariátrica e Metabólica. 2014. Sociedade Brasileira de Cirurgia Bariátrica e Metabólica alerta sobre a importância do pré-operatório para a segurança do paciente. Disponível em: https://www.sbcbm.org.br/ preparacao-eficiente-e-fundamental-para-a-realizacao-de-cirurgiabariatrica/. Acesso em: 10 jul. 2018.

BRASIL. Ministério da Saúde. Vigitel Brasil 2018: vigilância de fatores de risco e proteção para doenças crônicas por inquérito telefônico: estimativas sobre frequência e distribuição sociodemográfica de fatores de risco e proteção para doenças crônicas nas capitais dos 26 estados brasileiros e no Distrito Federal em 2018. Brasília: Ministério da Saúde, 2019. Disponível em: https://portalarquivos2.saude.gov.br/images/ pdf/2019/julho/25/vigitel-brasil 2018.pdf. Acesso em: 27 jan. 2020.

CARVALHO, T. S. de; VASCONCELOS, F. C. de; CARVALHO, M. D. B. M. Análise do Histórico de Métodos de Emagrecimento dos Pacientes Submetidos à Cirurgia Bariátrica em um Hospital Público de Belém-Pa. RBONE, São Paulo, v. 10, n. 55, p. 4-11, 2016.

COLLES, S. L.; DIXON, J. B.; O’BRIEN, P. E. Night eating syndrome and nocturnal snacking: association with obesity, binge eating and psychological distress. Int. J. Obes., London, v. 31, n. 11, p. 17221730, 2007.

GALLANT, A. R.; LUNDGREN, J.; DRAPEAU, V. The night-eating syndrome and obesity. Obes. Rer., Oxford, v. 13, n. 6, p. 528-536, 2012.

JASTRZĘBSKA-MIERZYŃSKA, M. et al. Dietary habits of obese patients qualified for bariatric procedures. Rocz Panstw Zakl Hig., Warszawa, v. 65, n. 1, p. 41-47, 2014.

JESUS, A. D. et al. Comportamento alimentar de pacientes de pré e pós-cirurgia bariátrica. RBONE, São Paulo, v. 11, n. 63, p. 187-196, 2017.

MITCHELL, J. E. et al. Eating behavior and eating disorders in adults prior to bariatric surgery. Int. J. Eat. Disord., New York, v. 48, n. 2, p. 215-222, 2015.

MONTEIRO, L. S. et al. Breakfast eating among Brazilian adolescents: Analysis of the National Dietary Survey 2008-2009. Rev. Nutri., Campinas, 30(4):463-476, jul./ago., 2017.

OVED, I. et al. Poor Health Behaviors Prior to Laparoscopic Sleeve Gastrectomy Surgery. Obes. Surg., Oxford, v. 27, n. 2, p. 469-475, 2017.

PEREIRA, M. A. et al. Breakfast Frequency and Quality May Affect Glycemia and Appetite in Adults and Children. J. Nutr., Philadelphia, v. 141, n. 1, p. 163-168, 2011.

RIBEIRO, G. A. N. A. et al. Depressão, ansiedade e compulsão alimentar antes e após cirurgia bariátrica: problemas que persistem. ABCD Arq. Bras. Cir. Dig., Curitiba, v. 31, n. 1, p. 1356, 2018.

RODRIGUES, L.G.; MATTOS, A. P.; KOIFMAN, S. Prevalence of metabolic syndrome in overweight and obese outpatient children and adolescents: comparative analysis using different clinical definitions. Rev. Paul. Pediatr., São Paulo, v. 29, n. 2, p. 178-185, 2011.

SILVA, T. S. L; CRAHIM, S. C. S. F. A importância da autoavaliação e acompanhamento psicológico para o paciente de cirurgia bariátrica. Revista Mosaico, Goiás, v. 10, n.1, p. 35-42, jan./jun., 2019.

UZHOVA, l. et al. Regularity of breakfast consumption and diet: insights from national adult nutrition survey. Nutrients., Suiça, v. 10, n. 11, p. E1578, 2018.

WORLD HEALTH ORGANIZATION (WHO). Obesity: preventing and managing the global epidemic. Geneva: World Health Organization, 2000.

WORLD HEALTH ORGANIZATION (WHO). Obesity and overweight. 2016. Disponível em: https://www.who.int/news-room/fact-sheets/detail/ obesity-and overweight. Acesso em: 28 jan. 2020. 